\title{
DETECTION OF CPFSK SIGNALS USING PER SURVIVOR PROCESSING
}

\author{
Mark J. Miller \\ ViaSat, Inc. \\ Carlsbad, CA
}

\begin{abstract}
The use of Per Survivor Processing (PSP) is considered in the detection of a Continuous Phase Frequency Shift keying (CPFSK) signal. It is shown that PSP can enable the use of a trellis structure for the demodulation that is different from the trellis structure used to generate the CPFSK signal. This allows one to select a receive trellis structure with fewer phases, resulting in a reduced state sequence estimation algorithm. Results are presented using a receive trellis of 3 states to receive binary CPFSK signal with a modulation index, $h$, of $7 / 10$. The complexity of the receiver is reduced by a factor of 3.3 with minimal performance degradation. An application of this PSP technique is also discussed which enables one to perform Maximum Likelihood Sequence Estimation (MLSE) of a digital FM signal, where the modulation index is only approximately known. Results presented for the binary case show this technique to provide a significant detection efficiency advantage over conventional techniques such as limiter-discriminator detection or non-coherent detection.
\end{abstract}

\section{INTRODUCTION}

Per Survivor Processing is a technique for providing an approximate Maximum Likelihood Sequence Estimation (MLSE) over channels where one or more parameters are not known. Typical applications include adaptive MLSE for channels with Inter Symbol Interference (ISI) and phase estimation in Trellis Coded Modulations (TCM) [1]. In this paper, PSP is used to provide an approximate MLSE receiver for CPFSK signals. This application of PSP results in a new reduced state sequence estimation algorithm for CPFSK signals as well as an approach to apply MLSE to digital FM signals.

Organization of this paper is as follows: Section II presents a brief review of CPFSK signals and maximum likelihood sequence estimation of such signals. Section III presents an overview of PSP and defines our use of this class of signal processing to CPFSK reception. Section IV presents our results, for the binary case, using PSP to yield a reduced state algorithm. Section $\mathrm{V}$ addresses the application of a PSP based receiver for the demodulation of a digital FM signal.

\section{CPFSK SIGNALS AND MAXIMUM LIKELIHOOD SEQUENCE ESTIMATION}

An M-ary CPFSK signal is defined in the time domain by [2],

$$
s(t, \bar{\alpha})=\sqrt{\frac{2 E}{T}} \cos (2 \pi f t+\phi(t, \bar{\alpha}))
$$

where $\bar{\alpha}$ is the data symbol sequence, $E$ is the energy per symbol, $T$ is the symbol duration, $f$ is the carrier center frequency, and $\phi(t, \bar{\alpha})$ is the information bearing phase which is defined over the $n^{\text {th }}$ symbol interval as,

$$
\phi(t, \bar{\alpha})=2 \pi h \sum_{j=-\infty}^{n} \alpha_{j} q(t-j T)
$$

Each data symbol, $\alpha_{j}$, in the sequence of data symbols $\bar{\alpha}$, takes on one of the $M$ values $\pm 1, \pm 3, \pm(M-1) . \quad M$ is generally equal to $2^{k}$, and thus $k$ input bits are used to specify one of $M$ M-ary symbols. For CPFSK the phase function, $q(t)$, is the well known 1REC pulse defined by

$$
q(t)=\frac{t}{2 T} \quad 0 \leq t<T
$$

with $q(t)=0$ for $t<0$ and $q(t)=1 / 2$ for $t \geq T$. Over the duration of the $n^{\text {th }}$ symbol, the carrier phase, $\phi(t, \bar{\alpha})$, changes linearly by an amount $\Delta \phi_{n}=\alpha_{n} h \pi$. The modulation index, $h$, is expressed as the ratio of two integers, $h=p / q$.

Selecting as a state variable the phase at the end of a symbol interval, one can construct a trellis for the carrier phase $\phi(t, \bar{\alpha})$. This trellis consists of $2 q$ states. Numbering the states from 0 to $2 q-1$, the relationship between the state number, $i$, and the phase corresponding to that state, $\theta^{i}$, is

$$
\theta^{i}=i \frac{\pi}{q}
$$

There are exactly $M$ branches or edges entering and exiting each state within the trellis. Defining the state of 
the transmitted signal at time $n$ (at the end of symbol $n$ ) as $S_{n}$, the next state, $S_{n+1}$, can be expressed as

$$
S_{n+1}=\left(S_{n}+p \alpha_{n+1}\right) \bmod 2 q
$$

and conversely, the previous state can be expressed from the current state

$$
S_{n-1}=\left(S_{n}-p \alpha_{n}\right) \bmod 2 q
$$

Equations (5) and (6) define the trellis with (4) providing the relationship between state number and carrier phase.

The maximum likelihood sequence estimation (MLSE) receiver of CPFSK signal operates by correlating the received signal against all possible paths through the trellis [2]. The symbol sequence, $\hat{\bar{\alpha}}$, that generated the path with the minimum squared Euclidean distance from the received signal becomes the output sequence. The realization of such a receiver uses $M$ filters, each matched to one of the $M$ possible frequencies, and the Viterbi algorithm.

The heart of the Viterbi algorithm involves the basic Add Compare Select (ACS) computation for updating the metrics and eliminating paths. Each state, at time $n$, has a metric, $M_{n}^{i}$, associated with it. For a given state $i$, let the $M$ possible previous states be $j 1$ through $j M$ which are defined by (6). The metric update equation for the new metric of state $i$ is,

$M_{n+1}^{i}=\max \left[M_{n}^{j 1}+B M_{j 1, i}, M_{n}^{j 2}+B M_{j 2, i}, \ldots, M_{n}^{j M}+B M_{j M, i}\right]$

where

$$
B M_{j, i}=\operatorname{Re}\left[z_{f(j, i)} e^{-j \theta^{j}}\right]
$$

In $(8), f(j, i)$ is the input data symbol that caused one to go from state $j$ to state $i$ in the trellis. The complex valued output of the filter matched to the data symbol $f(j, i)$ is denoted $z_{f(i, i)}$. After the ACS, the losing edges are eliminated and the bits corresponding to the winning edge are stored in the path history table. This process is performed for each of the states in the trellis. When $p$ is odd, only half the total number of states need to be updated each symbol, either the odd numbered states or the even numbered states.

When $p$ is even, only the even numbered states (assuming one started in an even numbered state) ever need to be updated. In both cases the total number of states that needed to be updated each symbol is $q$. Thus, the effective number of states in the trellis is $q$.

\section{PSP AND APPLICATIONS TO CPFSK RECEIVERS}

Per Survivor Processing was introduced as a technique for providing approximate Maximum Likelihood Sequence Estimation in an environment where some of the signal parameters are unknown. The concept behind it is that different estimates of the unknown parameters are associated with each state in the trellis. The estimates are updated from one node in the trellis to the next by using the data associated with the trellis edge in a data aided parameter estimation. At each node, only the estimate associated with the winning path to that node and the estimate associated with the state from which the winning path came are used to develop the new estimate to be associated with the state. This yields a per survivor estimation of the unknown parameters. Complete details of PSP can be found in [1].

Now consider the demodulation of an M-ary CPFSK signal using a trellis structure different than the trellis structure used to transmit the signals. Let the transmitted modulation index be $h_{t x}=p_{t x} / q_{t x}$. We shall detect this signal by using the quantity $h_{t x}$ to set the filter center frequencies but shall construct the trellis structure using the modulation index $h_{t x}=p_{t x} / q_{t x}$. Furthermore, let $h_{t x}$ and $h_{r x}$ be known and related to each other by $h_{t x}=h_{r x}+h_{\Delta}$. During symbol interval $n$, the actual phase of the signal will change by an amount

$$
\Delta \phi_{n}=\alpha_{n} h_{r x} \pi+\alpha_{n} h_{\Delta} \pi
$$

In the detection of the signal, the first term in (9) is tracked by the Viterbi algorithm that assumes a trellis structure based upon $h_{r x}$. The second term in (9) is the resulting phase difference which accumulates every symbol. This cumulative phase difference is the unknown quantity which will be estimated on a per survivor basis.

Using per survivor processing, we shall define an additional variable to be associated with each state. This variable, $\lambda_{n}^{i}$, will be the cumulative phase difference for state $i$ at time $n$ and is due to the accumulation of the second term in (9). This modifies our relationship between state number and phase as follows:

$$
\theta^{i}=\left(\lambda^{i}+i \frac{\pi}{q}\right) \bmod 2 \pi
$$

The receive trellis structure is still defined by (5) and (6) with $p$ and $q$ replaced by $p_{r x}$ and $q_{r x}$ respectively. The Viterbi algorithm proceeds as identified in section II with (10) used to provide the mapping between state and phase in the branch metric calculations. The only additional task 
that needs to be performed is the per survivor update of the cumulative phase difference for each state, $\lambda_{n}^{i}$. This is updated as follows:

$$
\lambda_{n+1}^{i}=\lambda_{n}^{j^{*}}+\alpha_{f\left(j^{*}, i\right)} h_{\Delta} \pi
$$

where $j^{*}$ is the previous state as selected in the maximization operation of (7) and $\alpha_{f\left(j^{*}, i\right)} \quad$ is the data symbol that caused one to go from state $j^{*}$ to $i$ in the receive trellis structure.

\section{REDUCED STATE SEQUENCE ESTIMATION}

Consider a binary CPFSK signal that is transmitted with a modulation index of $7 / 10$, a modulation index which maximizes the normalized minimum free distance. This modulation index results in a effective trellis size of 10 states. We shall consider receiving this signal using a modulation index, $h_{r x}$, of $2 / 3$. This results in a simple 3 state trellis. The resulting reduction in receiver complexity is a factor of 3.3 .

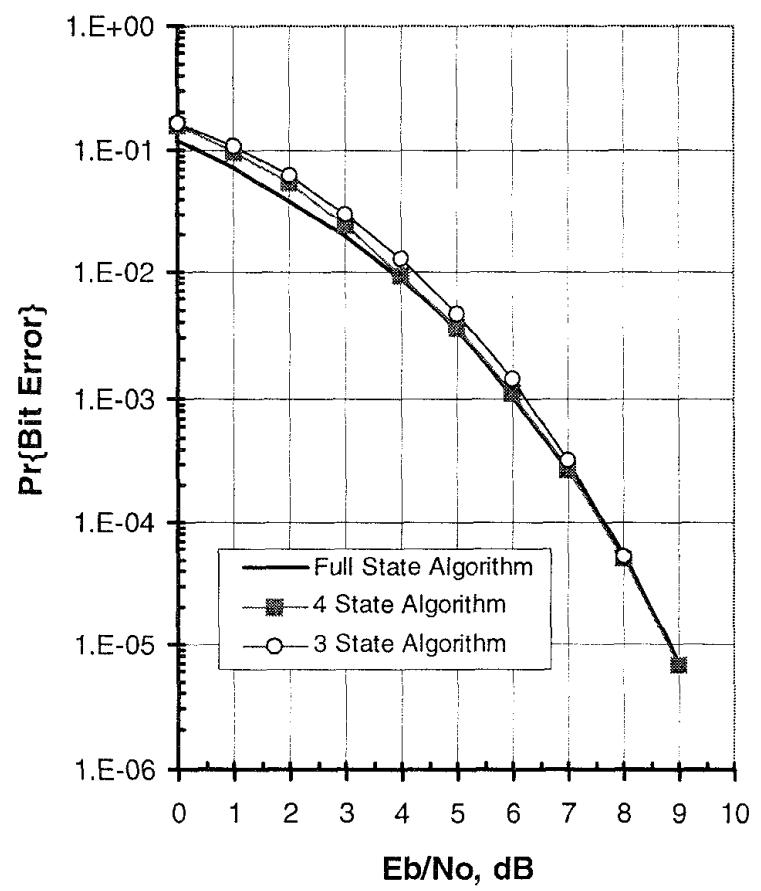

Figure 1. Comparison of Full State and Reduced State Performance

The resulting reduced state algorithm is a sub optimum receiver, hence Bit Error Rate (BER) bounds and approximations based upon the minimum distance of the transmitted signal are not valid. The performance of the reduced state algorithm has been determined by computer simulation with the results presented in Figure 1. As can be seen, the reduced state algorithm, based upon modulation index of $2 / 3$, results in insignificant performance degradation at BER's below $3 \times 10^{-4}$. At higher BER's, the performance degradation is small, $<1.0$ $\mathrm{dB}$. Using a 4 state, $h_{r x}=3 / 4$ based trellis for demodulation, the degradation is insignificant for BER's below $10^{-2}$. At higher BER's, the loss is about $0.5 \mathrm{~dB}$. In both cases, the slight deviation from theory at high BER's is due to an error propagation effect discussed below.

Certain error events will cause the cumulative phase difference term in the PSP processing to become incorrect for all future bits. This phase error in the cumulative phase difference terms will result in a higher BER that can propagate indefinitely. For example, consider the comparison of two different length 3 paths in the $h_{r x}=2 / 3$ based trellis. The first path (solid) is the 000 path and the second path (dash) is the 111 path. As shown in the trellis diagram in figure 2 , the paths leave state 0 and then remerge to state 0 after 3 symbols.

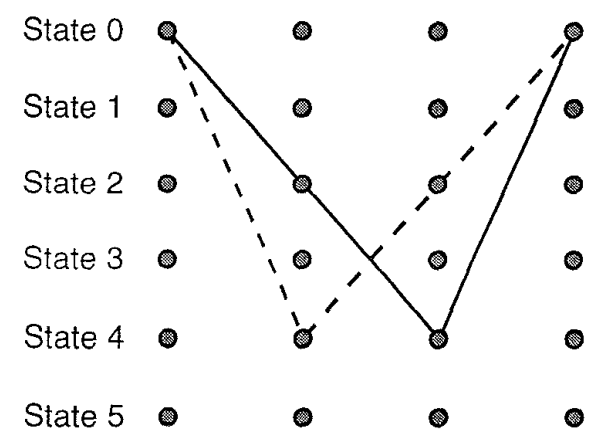

Figure 2. Trellis Paths for a Propagating Error

Let the first path be the correct path. If the correct path is chosen, the cumulative phase difference will increment by an amount $3 h_{\Delta} \pi$. But suppose, due to thermal noise, the incorrect path of 111 is chosen. The cumulative phase difference will be incremented by $-3 h_{\Delta} \pi$. The correct path will be eliminated and the correct cumulative phase difference will also be eliminated. The former results in the error event consisting of 3 bit errors but the later results in a cumulative phase difference error of $6 h_{\Delta} \pi$. This phase difference error will remain and affect the branch metric calculations for all future paths that originate from this state. If this phase difference error is large, and it usually is, this will cause the Viterbi algorithm to select paths that originate from another state which has a cumulative phase difference resulting in more favorable branch metrics. In general, this will still result 
in all future state phase values, $\theta^{i}$ being in error until another error event occurs which alters the cumulative phase difference in a manner as to correct the state phase values. This will cause a higher error rate to propagate until such an event occurs.

For binary CPFSK, the majority of the bit errors correspond to selecting the incorrect path when comparing paths that are separated by the normalized minimum squared Euclidean distance, $d_{\min }^{2}$. That corresponds with selecting a path of 01 over the correct path of 10 (or vice versa). This error event doesn't affect the cumulative phase difference, thus causing propagating errors, since the weight of both paths is the same. Propagating error events only occur when the weight of the two paths is different.

In practice, the impact of propagating error events can be made insignificant with the design of the appropriate carrier recovery loop. Such a loop should generate its phase error signal from within the Viterbi algorithm such that it sees the effect of the incorrect cumulative phase difference values. The effect of the carrier recovery loop is to limit the length of time the higher error rates can propagate. Our results shown in Figure 1 used second order carrier recovery loop. The results show the propagating error events to only have small impact on the BER which is only present at high BER's.

\section{MLSE OF DIGITAL FM SIGNALS}

A digital FM signal is a CPFSK signal that is generated by an analog FM modulator. A typical example would be a logic signal that takes on one of two voltages driving an analog FM modulator. In the case of a digital FM signal, the modulation index (or frequency deviation) used by the transmitter is never precisely known by the receiver, and furthermore, the modulation index may slowly drift with time. The receiver structure of such signals uses techniques that are insensitive to the precise value of the modulation index, such as limiter-discriminator detection [3] or non-coherent detection [4].

Maximum likelihood sequence estimation offers a significant performance improvement, but there are two obstacles that prevent it's use: 1) the modulation index is unknown, and 2) the modulation index may slowly vary over time. To support MLSE, the receive trellis structure (including the number of states) would have to be dynamically variable.
The PSP based receiver of CPFSK signals is the ideal mechanism to overcome the second obstacle. It allows the signal to be detected with a fixed trellis structure. Dynamic changes in the modulation index only affect the center frequency of the matched filters and the update of the cumulative phase difference as indicated in (11).

One still needs to accurately, but not exactly, determine the modulation index used during the transmission before our detection technique can be applied. This can be done by using circuitry or algorithms external to Viterbi Algorithm to estimate the modulation index. The estimation of this unknown parameter could also be performed within the PSP itself, a technique that has been shown to be particularly effective with other unknown channel parameters [1]. No matter what technique is used for estimating the unknown modulation index, there is still a residual error in the modulation index. The effect of this error on the demodulation performance is determined below.

Let the relationship between the transmit and receive modulation indices be $h_{t x}=h_{r x}+h_{\Delta}+h_{e}$, where $h_{e}$ now represents the error in the estimation of $h_{t x}$. When traversing through the correct path of the trellis, a phase error of $\varphi_{n}=\alpha_{n} h_{e} \pi$ is added to the cumulative phase difference each symbol. Since the transmitted symbols are independent, the phases errors that are added each symbol are independent with $E\left[\varphi_{n}\right]=0$ and variance

$$
E\left[\varphi_{n}^{2}\right]=\left(h_{e} \pi\right)^{2} \frac{2}{M} \sum_{i=1}^{M / 2}(2 i-1)^{2}
$$

This model is very similar to phase noise which is commonly modeled as a Wiener random process. Thus one would expect the problem of imperfect knowledge of the transmit modulation index to affect the performance of the receiver in the manner similar to phase noise. By adjusting the bandwidth of the carrier recovery loop, one can optimize receiver performance.

Performance and sensitivity to modulation index error has been determined by computer simulation for a binary CPFSK system. The modulation index used for transmission was $7 / 10+h_{e}$ and the received signal was detected using the trellis resulting from selection of $h_{r x}=3 / 4$. A second order carrier recovery loop was used with a damping ratio of 0.71 . The BER sensitivity to the modulation index error, $h_{e}$, is shown in figure 3 for a loop natural frequency of $f_{n}=R_{b} / 100$. Figure 4 shows the $\mathrm{E}_{\mathrm{b}} / \mathrm{N}_{\mathrm{o}}$ required for a BER of $10^{-4}$ as a function of modulation index for several different loop natural frequencies. 




Figure 3. Effects of Imperfect Knowledge of the Transmit Modulation Index

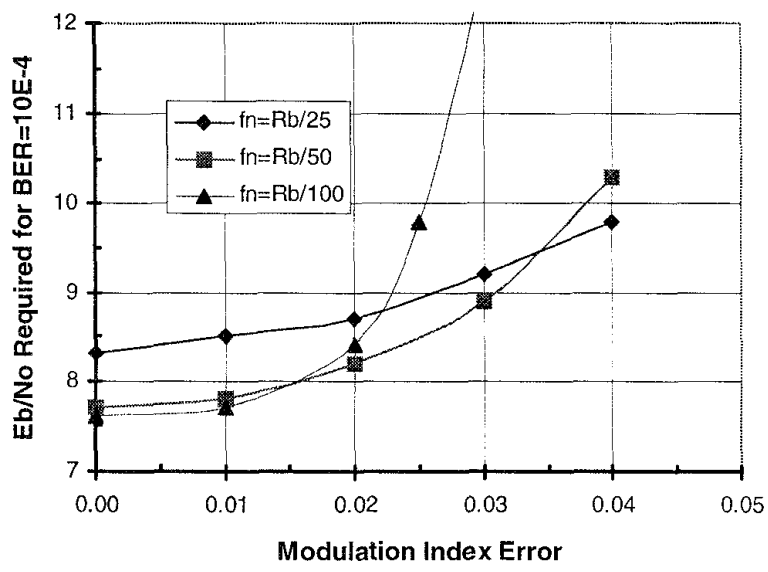

Figure 4. Required $E_{b} / N_{o}$ vs. Modulation Index Error

To illustrate the advantage of this digital FM receiver, figure 5 compares the detection efficiency of our near MLSE receiver with that of a limiter-discriminator receiver, and a non-coherent receiver. As illustrated, our near MLSE receiver offers a significant improvement in detection efficiency compared to conventional alternatives.

\section{SUMMARY}

We have introduced a new technique for detecting M-ary CPFSK signals which is a specific application of a more general class of processing termed Per Survivor Processing. This technique has been used to realize a reduced state MLSE receiver for CPFSK signals. Results have been presented for the binary case with minimal performance degradation and a significant reduction decoder complexity. We have also shown how to apply this technique to perform near MLSE of a digital FM signal. This resulting a detection efficiency is $3 \mathrm{~dB}$ better than that of conventional limiter discriminator detection and $6 \mathrm{~dB}$ better than non-coherent detection. The concepts presented in this paper are also extendible to partial response Continuous Phase Modulation (CPM), convolutional coded CPM, and multi- $h$ CPM modulations but have not yet been investigated by this author.

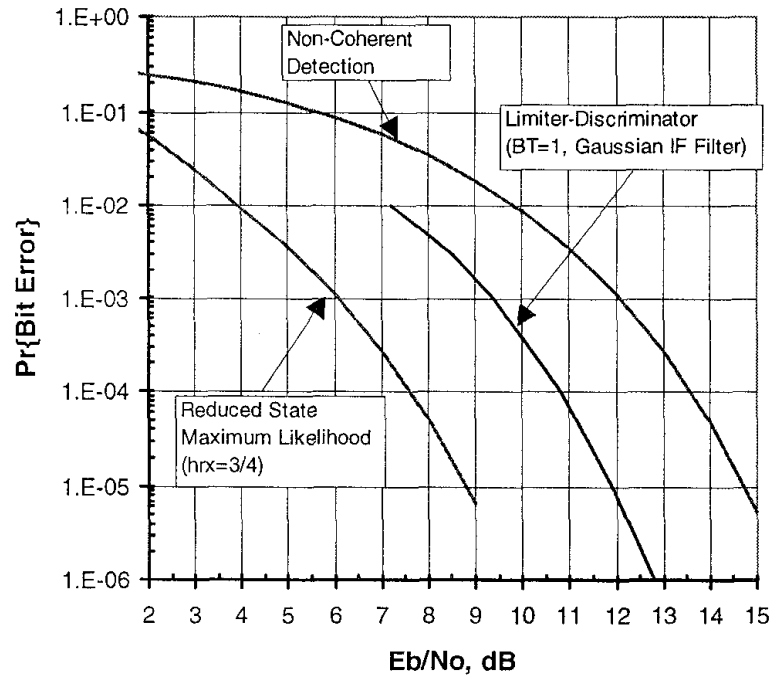

Figure 5. Comparison of Receiver Performance for Digital FM

\section{References}

[1] R. Raheli, A. Polydoros, C.K. Tzou, "Per-Survivor Processing: A General Approach to MLSE in Uncertain Environments", IEEE Transactions on Communications, Vol 43, No. 2/3/4, Feb/Mar/Apr 1995

[2] J.B. Anderson, T. Aulin, C.E. Sundberg, Digital Phase Modulation, Plenum Press, New York, NY,1986

[3] R.F. Pawula, "On the Theory of Error Rates for Narrow-Band Digital FM", IEEE Trans. On Communications, Vol. Com-29, No. 11, November 1981

[4] J.G. Proakis, Digital Communications, McGraw Hill Book Company, 1989 